\title{
Chapter 2 \\ Creating Effective Cultural Competence Workshops for Australian Higher Education Staff
}

\author{
Amy McHugh-Cole, Rachael Simons, and Gabrielle Russell
}

\begin{abstract}
Cultural competence, and Indigenous cultural competence in particular, is recognised as a priority in Australian higher education (Universities Australia, 2011). There is a need to develop the knowledge, skills and attitudes required to engage respectively and effectively in Aboriginal and Torres Strait Islander contexts in tertiary education settings across the country (Universities Australia, 2011). This chapter details a study conducted by the National Centre for Cultural Competence (NCCC), in its journey to embed cultural competence across a large higher education workforce. The study documents the creation and delivery of in-person workshops to determine the effectiveness of this approach in building understandings of, and commitment to, cultural competence among staff.
\end{abstract}

Keywords Cultural competence $\cdot$ Higher education $\cdot$ Aboriginal and torres strait islander $\cdot$ Pedagogy $\cdot$ Curriculum $\cdot$ Workshop facilitation

\section{Introduction}

In 2014, the National Centre for Cultural Competence (NCCC) was established at the University of Sydney. The focus of the NCCC is to lead the thinking on and practice of cultural competence in line with the University's Strategic Plan (The University of Sydney, 2016). Staff and students' capacity to work in a culturally competent manner is an organisational priority of the University (The University of Sydney, 2016). The Strategic Plan aligns with the seminal model put forward by Cross et al. (1989, p. 7), which positions cultural competence as a 'set of congruent behaviours, attitudes, and policies that come together in a system, agency, or amongst professionals and enables that system, agency, or those professionals to work effectively in crosscultural situations.' Kirmayer (2012) advocates that developing cultural competence at the organisational level and through the training and education of individual staff members is most effective. Cultural competence at the University is addressed at the strategic level, while the NCCC provides resources for University staff to develop

\footnotetext{
A. McHugh-Cole $(\varangle) \cdot$ R. Simons · G. Russell

National Centre for Cultural Competence, The University of Sydney, Sydney, NSW, Australia e-mail: amy.cole@sydney.edu.au 
their cultural competence capabilities at the individual level. This paper explores the journey taken by the NCCC to create and deliver those cultural competence workshops for staff.

The NCCC privileges Aboriginal and Torres Strait Islander knowledges, histories and contemporary realities when engaging University staff on the cultural competence journey. This aligns with the view of Universities Australia (2011, p. 17) that positions universities as agents of change, not only in improving higher education experiences and attainment for Indigenous Australians within universities but also by making 'a commitment to the capacity building of Indigenous communities' to reach more equitable outcomes for access and participation within these institutions.

In addition to prioritising Aboriginal and Torres Strait Islander contexts, the NCCC seeks to support and celebrate diversity at the University. The Australian Bureau of Statistics (2016) reports that $25.9 \%$ of the national population were born overseas. Nearly half of those who identify as Australian (49\%) were either born overseas or have one or both parents who were born overseas (Australian Bureau of Statistics, 2017). This data suggests that individuals from different cultural backgrounds will come into contact with one another, whether it be in the communities where they live, the organisations in which they work or the institutions in which they study.

As cultural diversity increases, an overwhelming majority of Australians perceive multiculturalism positively (Markus, 2017). Nonetheless, racism and racial discrimination continue to be an issue for many in the community. According to the Scanlon Foundation's Mapping Social Cohesion project (Markus, 2017), 34.2\% of people surveyed from non-English speaking backgrounds had experienced discrimination in the past twelve months. Aboriginal and Torres Strait Islander people experience individual and systemic racism at higher levels still. According to the Reconciliation Barometer, 33\% of Indigenous respondents reported experiencing verbal racial abuse in the previous six months (Reconciliation Australia, 2018). A commitment to combatting racism and developing cultural competence in community, workplace and university settings is imperative.

Staff at the University represent a diverse workforce comprising more than 100 different countries of home origin (personal communication, Mery Joseph, January 29,2018 ). Approximately $36 \%$ of the University's student body is made up of individuals who have come to study at the University from more than 175 different countries of home origin (The University of Sydney, 2018). The University prioritises developing cultural competence in its staff and graduates, advocating that the 'organisational culture must enable each member of the University to thrive and realise their full potential' (The University of Sydney, 2016, p. 43). Cultural competence, when embedded throughout an entire system, can provide for safer, respectful and more supportive workplaces and learning environments. University staff require educational resources and tools in order to feel capable and supported in their efforts to infuse cultural competence into their context. 


\section{The NCCC's Approach to Cultural Competence Pedagogy}

The NCCC aims to develop individual cultural competence capabilities whilst at the same time equipping leaders to address necessary organisational change. The NCCC's resources include a series of online modules, a massive open online course (MOOC), leadership programmes and face-to-face workshops. The in-person workshops for staff, which will be the focus of this paper, build on the information in the online modules to assist staff to develop their cultural competence capabilities. This is referred to as blended learning.

Taking a blended learning approach allows for a fusion of face-to-face and online learning experiences (Garrison \& Vaughan, 2007) and provides participants with opportunities to learn both on their own and in a supportive face-to-face environment with colleagues. It also gives the NCCC facilitators the opportunity to disseminate some foundational information in the online environment, allowing for more interactive learning in the face-to-face workshops.

Blended learning also supports the NCCC's position that critical self-reflection is an important part of the journey towards cultural competence (Sherwood \& RussellMundine, 2017). Asking participants to undertake the online modules prior to attending an in-person workshop allows time to process material and develop the skills that are necessary to critically self-reflect during and after the in-person workshops. Indeed, it is imperative for individuals to begin the cultural competence journey by reflecting on their own cultures and the ways in which these influence how they think, act and behave, before trying to understand another culture (Ranzijn McConnochie, \& Nolan, 2009).

\section{Workshop Creation}

The development of the workshops was through a collaborative approach involving NCCC academics. In creating the resources, we modelled critical self-reflection, which is a fundamental capability for effective cultural competence. Built into the development process was a critical reflection cycle incorporating feedback and selfobservation (Sherwood \& Russell-Mundine, 2017).

Once the first iteration of each workshop was produced, pilot workshops were facilitated for colleagues within the Deputy Vice-Chancellor (Indigenous Strategy and Services) portfolio and other members and friends of the NCCC network. Participants provided formal feedback after each of the four pilot workshops in the form of survey responses in addition to feedback provided anecdotally during the sessions. The feedback was used to adjust and refine the program before making the workshops available to all University staff. When the first workshops were delivered the online modules were still in development, so each workshop was a full day in length. Following the release of the online modules and in response to feedback that fullday workshops were hard to commit to, workshops were reworked for delivery in a 
half-day format. Completion of the first two online modules on social and emotional wellbeing and the fundamentals of cultural competence became a pre-requisite for attending the workshops.

\section{Workshop Structure}

Developing cultural competence requires understanding a number of core components. These include understanding socialisation and worldview, one's cultural identities and critical self-reflection (National Centre for Cultural Competence, 2016). These concepts are scaffolded throughout the workshops. Each workshop has between two and four sessions that include learning outcomes, facilitator input and activities designed to support the learning outcomes and develop participants' cultural competence capabilities. There is emphasis that the journey towards cultural competence is lifelong and requires a commitment by the participants to constantly recognise and challenge their biases and assumptions.

The four foundational workshops offered by the NCCC are: (1) Cultural Competence: Social and Emotional Wellbeing; (2) Cultural Competence: The Fundamentals; (3) Cultural Competence: Relational Learning; and (4) Cultural Competence: The Foundations of Racism. The workshop on social and emotional wellbeing was designed as the starting point for University staff's journeys towards cultural competence. Drawing on an Indigenous framework, the workshop highlighted the link between having a strong sense of identity and overall wellbeing. Participants learned about identity and why it is important to understand oneself before trying to understand others and developed their resiliency skills in the context of their cultural competence journey. The second workshop introduced participants to the building blocks of cultural competence. Participants gained foundational knowledge, learned about the impact of cultural incompetence in social institutions such as higher education and developed skills to infuse cultural competence pedagogies and resources into their work.

The relational learning workshop provided teaching staff (including sessional staff) with the knowledge and resources to shift their teaching approach to facilitate student learning through a relational epistemology. Participants learned about their inter-relatedness and interdependence with each other and their broader social, political, cultural, environmental and professional contexts. Finally, the workshop on the foundations of racism introduced participants to the historical context and contemporary forms of racism in Australia and outlined the role that cultural competence can play in combating racism. Participants deepened their understanding of interpersonal and institutional racism and developed their ability to recognise, acknowledge and challenge racism when they encounter it within their work environment.

While each workshop focuses on a different topic, the overall structure of each workshop is consistent, and the content is scaffolded. Every workshop begins with an acknowledgment of country, overview of the workshop, work health and safety information, facilitator and participant introductions, and includes time for participants 
to write their expectations for the workshop. Participants are then introduced to the principles for engagement, in which the NCCC's overarching engagement principles are set out, discussed and agreed upon before the workshop activities commence.

\section{Methodology}

The updated in-person staff workshops were offered on a continuing basis to all University staff beginning in Semester 2, 2016. After attending an in-person workshop, participants were invited to fill out online surveys and/or participate in an in-person focus group. Participation was voluntary, and ethics approval for this research was sought and granted by the University's ethics committee. Survey data was collected from offerings of the four workshops between 2017 and 2019. A mixed-methods approach was used, asking participants to answer multiple choice, matrix/scale rating and open-ended questions. Descriptive statistics and thematic analysis were applied to analyse the data, and the information was utilised to help make improvements to the workshops. Qualitative data from a focus group held in 2016 was also included as a separate category for analysis. The voluntary nature of the workshops, and of participation in this study, resulted in a low response rate. However, data received was found to be useful in determining the effectiveness of the workshops and providing insights into particular strengths and areas for improvement going forward.

\section{Data/Results}

\section{Online Surveys}

In the social and emotional wellbeing workshop, $100 \%(n=11)$ of respondents felt that initial information on the topic was provided in an effective or highly effective manner (Fig. 2.1). This suggests that participants came to the workshop with little

Fig. 2.1 Workshop effectiveness-social and emotional wellbeing

\section{How effective was this workshop in providing initial information about social and emotional wellbeing?}

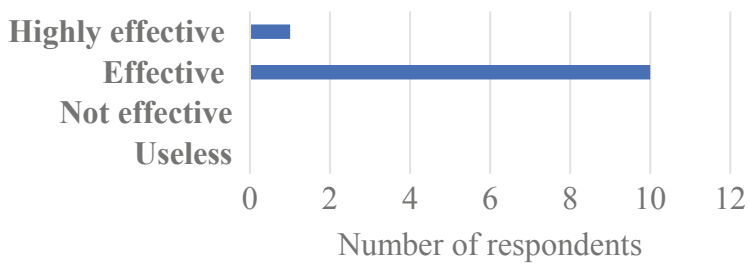


Fig. 2.2 Workshop effectiveness-cultural competence capabilities

\section{How effective was this workshop in helping you understand more about your own cultural competence capabilities?}

\section{Highly effective \\ Effective \\ Not effective \\ Useless}

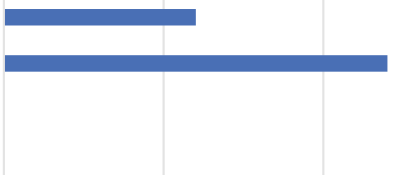

0

10

15

Number of respondents

prior understanding of the concepts covered, and thus the introductory nature of the workshop was appropriate. Likewise, all respondents felt the workshop effectively helped them understand more about their own social and emotional wellbeing. After the workshop, the majority of respondents $(n=9)$ felt equipped with ideas about how to address social and emotional wellbeing in their local work context. The two neutral responses suggest that this is an area where discussion could be more effectively framed to ensure sharing of ideas among participants can help to facilitate the implementation of social and emotional wellbeing principles within the various work contexts of the University.

Similarly, those who attended the fundamentals of cultural competence workshop felt that initial information was provided effectively (highly effective: $n=10$; effective: $n=9$ ). When asked to evaluate the effectiveness of the workshop in helping them understand their personal cultural competence capabilities (Fig. 2.2), responses were positive (highly effective: $n=6$; effective: $n=12$ ). This was similarly expressed by participant's feeling equipped with information to implement cultural competence into their work (highly effective: $n=5$; effective: $n=13$ ). In the open-ended question regarding the most helpful component of the workshop, active listening and worldview activities emerged as common themes.

Analysis of feedback from the relational learning workshop provides insight into the effectiveness of the approach to teaching a pedagogical component of cultural competence. Of the 11 responses received, $100 \%$ reported that the topic was presented in a logical manner. As a topic that can be unfamiliar to staff, ensuring logical presentation and structuring of the workshop was imperative. The repeated reference to interpersonal discussions and activities as a helpful aspect of the workshop is in itself reflective of a success of the approach used, with dialogue and engaging with others being an important component of relational learning.

Feedback from the foundations of racism workshop provides important insights not only into the effectiveness of the content and facilitation but also into how racism might be experienced within and beyond the university context. All respondents $(n=12)$ found the workshop effective in providing foundational information about racism. When asked whether participants felt capable to discuss the ideas surrounding racism with their colleagues (Fig. 2.3), positive responses (strongly agree: $n=4$; 
Fig. 2.3 Workshop effectiveness-racism discussion
I feel capable to discuss the ideas surrounding racism with my colleagues.

\author{
Strongly agree \\ Agree \\ Neutral \\ Disagree \\ Strongly disagree
}

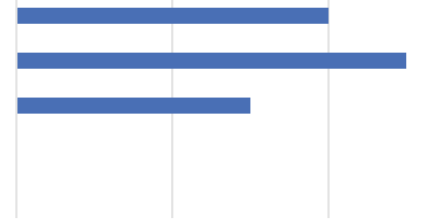

0

agree: $n=5)$ were received alongside neutral responses $(n=3)$. This suggests that racism continues to permeate the higher education context and remains an area of concern for staff who seek more practical ways to engage in anti-racism work through this training.

\section{Focus Group}

A focus group was held after our first round of workshops in 2016. Three participants attended in person, while two additional participants answered the same questions via email. Two of the authors of this chapter conducted the focus group that lasted approximately $45 \mathrm{~min}$. Participants were asked a number of questions related to their experience(s) of our in-person workshops, ways in which they've continued their cultural competence journeys and recommendations for improvement.

\section{Themes}

A thematic analysis of the data revealed four themes, which were (a) a desire for continued connections, (b) effectiveness, (c) a need for clarity and (d) recommendations.

\section{a. A desire for continued connections}

Connectedness was identified as particularly important for participants. Some participants indicated that after they finished the workshop(s), the journey seemed to halt for them. As one participant articulated:

I really like (being) connected and would like to carry that conversation on. And you think you're going to when you walk out there, but you get back to your desk and there's emails and things to do, and then the PowerPoint gets filed there, and the workbook gets put down there.

Participants expressed that they had enjoyed the workshops and wanted to continue the collaborative journey with fellow participants after its conclusion. There was agreement that if communication between workshop participants could be continued, their cultural competence journey could progress more effectively. One participant 
expressed that 'it would be better for me in kind of keeping it all going if the relationships kept going.' This idea that connections to colleagues could assist in motivating participants along their cultural competence journey speaks to the need for a systems approach:

I see the whole thing as trying to move the University's attention to these things more to centre stage, and I guess for me that will happen if people keep networking and will also happen if we can see the connection between what's happening in gender studies, what's happening in student admin, you know that kind of thing?

\section{b. Effectiveness}

Another theme that emerged was effectiveness. Within this theme, two of the subsections (self-reflection and peer interaction) speak to the content and structure of the in-person workshops. The third sub-section (impact on work) speaks to the follow-on effect for the participants attending one or more of the workshops.

\section{- Self-reflection and peer interaction}

An important component of cultural competence is critical self-reflection. To develop cultural competence, one needs to interrogate one's knowledge, how that knowledge was created and how it affects their interactions with others (Cooper, He, \& Levin, 2011). Walker, Schultz and Sonn (2014, p. 200) put forward that 'cultural competence is the ability to identify and challenge one's own cultural assumptions, values and beliefs. It is about developing empathy and connected knowledge, the ability to see the world through another's eyes, or at the very least to recognise that others may view the world through a different cultural lens.' This is why self-reflection features heavily in all NCCC teaching and learning resources. A number of participants mentioned that self-reflection was a welcome part of the workshops, 'for me, [it] seemed like a good first step and it does feel like you're doing it in quite a self-reflective way.'

When asked which feature, activity or interaction in the workshop was the most helpful, a number of respondents indicated that the guided self-reflection was a powerful tool. One participant specifically noted: 'guided self-reflection and hearing stories of other members' self-reflection too clearly demonstrated the depth and complexities around what influences people's actions and perceptions.' Exposure to the worldview of others assists in moving us along our cultural competence journey. Our worldview is what guides our decision making and what helps us to make sense of the world. Behaviours and actions can stem from ones' worldview, which is why it is important to connect with others to try and learn more about where their worldview comes from (Ranzijn et al., 2009).

The workshops gave participants the opportunity to extend understanding through interactions with others: 'They [the workshops] complemented the online modules and gave me the opportunity to have very stimulating conversations and enhance my learning through those interactions.' When asked which feature, activity or interaction in the workshop was the most helpful, participants referenced interaction and exposure to others' worldviews: 'the opportunity to extend understanding through discussion and exposure to different perspectives, experiences and understandings'; 
'diverse conversations and different lenses that people used'; 'I so enjoyed the conversations that I've had with people here, during the workshops. And probably in ways that I never would have in a casual meeting.'

\section{- Impact on work}

Following participation in one or more workshop, respondents noted the impacts on the workplace: 'I've worked to embed cultural competence within the Library as a staff capability, but also as something that informs our delivery of spaces and services to be inclusive, accessible and sensitive, as articulated in our Library Strategic Plan.' Another participant remarked 'I've tried to embed material [from the workshop] in my first-year course.' Others found that participation in the workshops helped to make once abstract concepts more concrete and visible in the workplace, commenting, 'then you kind of go about your day and you see examples it or you feel examples of it that you hadn't necessarily noticed before because you didn't have the tools or the language around it.'

\section{c. A need for clarity}

Another theme that came through was participants' desire for clarity. As the NCCC worked to create and disseminate the workshops as a component of the suite of resources, the way those resources 'fit together' seemed confusing for those outside of the NCCC. One participant commented, 'I just felt like the way it was presented I couldn't actually work out how the four courses fitted [sic] together.' There also seemed to be confusion around who the workshops were meant for. Professional and academic staff were encouraged to attend, but this was unclear to participants, with it being remarked, 'I was a bit like 'Is that actually something I can go to?' This was expressed by another participant:

I looked at the other ones and they looked a little bit abstract or a little bit ambiguous - I'm glad you used that word. Because I was a bit like 'Oh why do they apply to me? I don't really get that.' So, I didn't really know how they fit it into the online modules as well.

\section{d. Recommendations}

Our focus group and survey respondents shared a number of recommendations with us, many of which have since been implemented. One of the most salient recommendations was participants' interest in having a clear connection between our workshops (how they fit together, why the specific topics), and how the workshops then fit in with the suite of resources overall.

Respondents also recommended that the online modules and in-person workshops be combined in some way. From this recommendation, some content was removed from the Cultural Competence: The Fundamentals workshop that was covered in the first two online modules, and then made completion of the first two online modules a pre-requisite for attendance at any of the face-to-face workshops. This has helped to allay another issue, which was that participants would come to the sessions with varying levels of understanding about cultural competence. Requiring participants to complete the online modules before attending in person ensured that participants were on the same base-line level from which to continue their journey. 
Another important recommendation was to further utilise the strategic plan and graduate attributes. The University's 2016-20 Strategic Plan calls on the campus community to embed cultural competence into the culture of the University, as well as into the student curriculum. This recommendation reminded us that not everyone on campus may be aware of the strategic plan or what is included in it. Its promotion as a tool for participants to utilise as they continue their cultural competence journey is thus imperative, as the systems-level support is what will enable the embedding of cultural competence into all aspects of the University.

\section{Limitations}

The small sample size is a limitation of this study. This limitation can be explained by the voluntary nature of participation in the workshops and research study. Going forward, longitudinal research should be undertaken to assess workshop effectiveness over time and within various contexts across campus (academic and staff spaces).

\section{Conclusion}

This study sought to determine the effectiveness of workshops in the development of staff cultural competence at an Australian higher education institution. Analysis of the survey and focus group data found that the workshops were effective in educating participants about various aspects of cultural competence, including social and emotional wellbeing, the fundamentals of cultural competence, relational learning and the foundations of racism. Our workshops do not seek to provide a 'tick-a-box' solution. The findings of this research leave us optimistic that the NCCC resources are forming an integral part of driving cultural change within this tertiary setting.

\section{References}

Australian Bureau of Statistics. (2016). Data by region. Retrieved from http://stat.abs.gov.au/itt/r. jsp?databyregion\&ref=CTA2\#/.

Australian Bureau of Statistics. (2017). 'Census reveals a fast changing, culturally diverse nation' (Media Release, 27 June 2017). Retrieved from http://www.abs.gov.au/ausstats/abs@.nsf/lookup/ Media\%20Release3.

Australian Government-Department of Home Affairs. (2017). Multicultural Australia: United, Strong, Successful. Retrieved from https://www.homeaffairs.gov.au/LifeinAustralia/Documents/ MulticulturalAffairs/english-multicultural-statement.pdf.

Cooper, J. E., He, Y., \& Levin, B. B. (2011). Who I am: How can we understand ourselves as cultural beings? In Developing Critical Cultural Competence: A Guide for 21 Century Educators. Thousand Oaks, California: Corwin Press. 
Cross, T., Bazron, B. J., Dennis, K. W., \& Isaacs, M. R. (1989). Towards a Culturally Competent System of Care: A monograph on effective services for minority children who are severely emotionally disturbed. Washington DC: CASSP Technical Assistance Center, Georgetown University Child Development Center.

Garrison, D. R., \& Vaughan, N. D. (2007). Blended learning in higher education. San Francisco: Jossey-Bass.

Kirmayer, L. J. (2012). Rethinking cultural competence. Transcultural Psychiatry, 49(2), 149-164.

Markus, A. (2017). Mapping Social Cohesion: The Scanlon Foundation surveys 2017. Retrieved from http://scanlonfoundation.org.au/socialcohesion2017/.

National Centre for Cultural Competence. (2016). What is cultural competence? Retrieved from https://sydney.edu.au/nccc/about-us/what-is-cultural-competence.html.

Ranzijn, R., McConnochie, K., \& Nolan, W. (2009). Psychology and Indigenous Australians: foundations of cultural competence. Victoria: Palgrave Macmillan.

Reconciliation Australia. (2018). Australian Reconciliation Barometer 2018. Retrieved from https://www.reconciliation.org.au/wp-content/uploads/2019/02/ra_2019-barometer-brochure_ web.single.page_.pdf.

Sherwood, J., \& Russell-Mundine, G. (2017). How we do business: Setting the agenda for cultural competence at the University of Sydney. In J. Frawley, S. Larkin, \& J. A. Smith (Eds.), Indigenous pathways, transitions and participation in higher education: From policy to practice (pp. 133150). Singapore: Springer Open.

The University of Sydney. (2018). Enrolment countries. Sydney, NSW: The University of Sydney.

The University of Sydney. (2016). 2016-20 Strategic Plan. Retrieved from https://sydney.edu.au/ about-us/vision-and-values/strategy.html.

The University of Sydney. (2012). Wingara Mura-Bunga Barrabuggu: The University of Sydney Aboriginal and Torres Strait Islander Integrated Strategy. Retrieved from http://sydney.edu.au/ strategy/docs/wingara-mura-bunga-barrabugu.pdf.

Universities Australia. (2011). National Best Practice Framework for Indigenous Cultural Competency in Australian Universities. Retrieved from http://www.indigenousculturalcompetency.edu. au/index.html.

Walker, R., Schultz, C., \& Sonn, C. (2014). Cultural Competence-Transforming Policy, Services, Programs and Practice. In P. Dudgeon, H. Milroy, \& R. Walker (Eds.), Working together: Aboriginal and Torres Strait Islander mental health and wellbeing principles and practice. Australian Institute of Health and Welfare: Canberra, ACT.

Open Access This chapter is licensed under the terms of the Creative Commons Attribution 4.0 International License (http://creativecommons.org/licenses/by/4.0/), which permits use, sharing, adaptation, distribution and reproduction in any medium or format, as long as you give appropriate credit to the original author(s) and the source, provide a link to the Creative Commons license and indicate if changes were made.

The images or other third party material in this chapter are included in the chapter's Creative Commons license, unless indicated otherwise in a credit line to the material. If material is not included in the chapter's Creative Commons license and your intended use is not permitted by statutory regulation or exceeds the permitted use, you will need to obtain permission directly from the copyright holder.

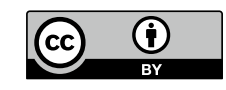

